\title{
Relationships between Mechanical Variables in the Traditional and Close-Grip Bench Press
}

\author{
by \\ Robert G. Lockie', Samuel J. Callaghan', Matthew R. Moreno ${ }^{3}$, Fabrice G. Risso', \\ Tricia M. Liü, Alyssa A. Stage 3 , Samantha A. Birmingham-Babauta ${ }^{3}$, \\ John J. Stokes 3 , Dominic V. Giuliano ${ }^{3}$, Adrina Lazar ${ }^{3}$, DeShaun L. Davis ${ }^{3}$, \\ Ashley J. Orjalo ${ }^{1}$
}

The study aim was to determine relationships between mechanical variables in the one-repetition maximum (1RM) traditional bench press (TBP) and close-grip bench press (CGBP). Twenty resistance-trained men completed a TBP and CGBP 1RM. The TBP was performed with the preferred grip; the CGBP with a grip width of 95\% biacromial distance. A linear position transducer measured: lift distance and duration; work; and peak and mean power, velocity, and force. Paired samples $t$-tests $(p<0.05)$ compared the 1RM and mechanical variables for the TBP and CGBP; effect sizes $(d)$ were also calculated. Pearson's correlations $(r ; p<0.05)$ computed relationships between the TBP and CGBP. $1 R M$, lift duration, and mean force were greater in the TBP $(d=0.30-3.20)$. Peak power and velocity was greater for the CGBP $(d=0.50-1.29)$. The 1RM TBP correlated with CGBP 1RM, power, and force $(r=0.685-0.982)$. TBP work correlated with CGBP 1RM, lift distance, power, force, and work $(r=0.542-0.931)$. TBP power correlated with CGBP $1 R M$, power, force, velocity, and work $(r=0.484-0.704)$. TBP peak and mean force related to CGBP 1RM, power, and force $(r=0.596-0.980)$. Due to relationships between the load, work, power, and force for the TBP and CGBP, the CGBP could provide similar strength adaptations to the TBP with long-term use. The velocity profile for the CGBP was different to that of the TBP. The CGBP could be used specifically to improve high-velocity, upper-body pushing movements.

Key words: 1RM, bar velocity, force, linear position transducer, power, upper-body strength.

\section{Introduction}

The traditional bench press (TBP) is commonly used to develop upper-body pushing strength (Golas et al., 2017; Gomo and Van Den Tillaar, 2016; Lehman, 2005; Stastny et al., 2017). This exercise involves the individual lying supine on a bench, and lowering an Olympic bar towards the chest, before forcefully pressing the bar away from the chest via arm extension (Gomo and Van Den Tillaar, 2016). The grip position adopted in the TBP is usually a preferred width where the individual feels they can lift the greatest load (i.e. the individual's most powerful position) (Lockie et al., 2017). This will generally result in a hand position on the bar that is wider than the shoulders, which means that when the bar contacts the chest, the elbows form an approximate $90^{\circ}$ angle at the bottom position (Clemons and Aaron, 1997). This results in a TBP

1 - Department of Kinesiology, California State University, Fullerton, Fullerton, USA.

2 - Centre for Exercise and Sport Science, School of Exercise and Health Sciences, Edith Cowan University, Joondalup, Australia.

3 - Department of Kinesiology, California State University, Northridge, Northridge, USA. 
grip width that will typically fall within a range of $165-200 \%$ of biacromial distance (BAD) (Wagner et al., 1992). However, there are potential issues with grip widths at the end of the range documented by Wagner et al. (1992). A TBP performed with a grip width of $\geq 200 \%$ BAD can place the shoulder joint in $90^{\circ}$ of abduction (Green and Comfort, 2007), which is an 'at-risk' position for injuries (Gross et al., 1993). As a result, the use of the TBP with a wider grip could be problematic for those individuals who experience shoulder issues. Furthermore, strength adaptations to an exercise tend to be joint angle-specific (Folland et al., 2005; Kitai and Sale, 1989). The hand position adopted in the TBP may not result in the most optimal crossover to hand and arm positions required in sports.

The TBP can be modified to adjust how the shoulder joint is positioned in the exercise. Indeed, grip widths of $\leq 150 \%$ BAD have been recommended for the bench press exercise as they may decrease the risk of injury (Green and Comfort, 2007; Haupt, 2001). The close-grip bench press (CGBP) is one variation where the grip width is reduced, typically to a range of $95-100 \%$ BAD (Clemons and Aaron, 1997; Lehman, 2005; Lockie et al., 2017; Wagner et al., 1992). This hand position leads to less shoulder abduction, which could reduce the stress placed upon this joint (Green and Comfort, 2007; Haupt, 2001). Further to this, even though the muscles in the chest and anterior deltoid region are still recruited, the close-grip bench press can place greater emphasis on the triceps (Barnett et al., 1995; Clemons and Aaron, 1997; Lehman, 2005). Additionally, reduced shoulder abduction keeps the hands close to the torso throughout the CGBP (Gomo and Van Den Tillaar, 2016).

The change in the hand position could lead to specific strength training adaptations following the chronic use of this exercise. This is pertinent, because as previously stated, strength adaptations are specific to the joint angles utilized during training (Folland et al., 2005; Kitai and Sale, 1989). If the TBP is the predominant upperbody pressing exercise used within a training program, then strength will be developed specific to a wider hand position. This is despite the fact that many sports involve pushing actions where the hands are positioned closer to the frame of the torso. Indeed, this hand position relates to the pushing actions required in American football for offensive linemen involved in run and pass blocking (Stokes et al., 2010), fending in rugby union and rugby league (Wheeler and Sayers, 2011), and performing a chest pass in basketball (Delextrat and Cohen, 2009) and netball (Cronin and Owen, 2004). The CGBP could be used as an alternate bench press exercise to develop strength specific to actions required in sport, in addition to those who experience shoulder issues when performing the TBP. However, there has been little analysis of this exercise. Greater understanding of the mechanics of this exercise, including the power, force, velocity, and work characteristics, may encourage greater usage of the CGBP. If these mechanical variables are relatable to those from the TBP, strength and conditioning coaches may more consistently program the CGBP as it more closely mirrors the upper-body actions required in many sports.

Therefore, this study investigated the relationships between mechanical variables (lift distance and duration, work, peak and mean power, force, and velocity) for the TBP and CGBP. This determined whether there were similarities between the two exercises, and provided practitioners with the knowledge whether the CGBP could train similar qualities (e.g. movement velocity, power and force output) as the TBP, while utilizing a hand position that is closer to the torso frame and more similar to athletic movements. It is important to investigate the relationships between the different mechanical variables in the TBP and CGBP, as any changes in lift distance and duration can influence the resulting work, power, force, and velocity generation (Lockie et al., 2017). Furthermore, no scientific study has analyzed relationships between the mechanics of the TBP and CGBP, despite their use in resistance training programs (Green and Comfort, 2007; Lockie et al., 2017), and thus it is important to establish actual relationships between these two lifts rather than relying on assumptions. Accordingly, 20 trained men performed one-repetition maximum (1RM) lifts for both the TBP and CGBP, and each lift was measured with a linear position transducer. The use of a linear position transducer to measure the TBP and CGBP ensured the data would be useful for practitioners, due to the use of this equipment in the field (Drinkwater et al., 2007; Harris et al., 
2010; Lockie et al., 2017; Lockie et al., in press). The data recorded for this study included: lift distance (i.e. bar displacement) and duration; work; and peak and mean power, velocity, and force. It was hypothesized that there would be significant differences in the load and the mechanical variables between the TBP and CGBP. It was further hypothesized that these variables for the TBP would correlate with those from the CGBP.

\section{Methods}

\section{Participants}

Twenty men (age $=24.05 \pm 4.42$ years; body height $=1.75 \pm 0.06$ meters $[\mathrm{m}]$; body mass $=$ $81.32 \pm 16.07$ kilograms [kg]) volunteered to participate in this study. Participants were recruited from the student population at the university via information sessions on campus and word-of-mouth amongst the students. Prior to participant recruitment, G*Power software (v3.1.9.2, Universität Kiel, Germany) was used to confirm that the sample size of 20 was sufficient for a paired-samples t-test analysis, and ensured the data could be interpreted with a moderate effect level of 0.58 (Hopkins, 2004), and a power of 0.80 when significance was set at 0.05 (Faul et al., 2007). Furthermore, $G^{*}$ Power software confirmed that this sample size was sufficient for a correlation, point biserial model, and ensured the data could be interpreted with a moderate effect level of 0.56 (Hopkins, 2004), and a power level of 0.80 when significance was set at 0.05 (Faul et al., 2007). The calculations and process used for these statistical variables have been provided with detail by Faul et al. (2007). All participants were required to: be currently resistance training ( $\geq$ three hours per week); have a resistance training history ( $\geq$ two times per week) of at least two years; be experienced with completing maximal lifts, including the TBP and CGBP; and free from any musculoskeletal disorders. The institutional ethics committee approved the procedures, all participants received a clear explanation of the study, including the risks and benefits of participation, and written informed consent was obtained.

\section{Design and Procedures}

One testing session was used per participant, and all assessments were conducted in the teaching gym at the university. Prior to data collection, the participant's age, body height, body mass, and BAD were recorded. Body height was measured barefoot using a portable stadiometer (seca, Hamburg, Germany), while body mass was recorded by electronic digital scales (Tanita Corporation, Tokyo, Japan). BAD was measured as the distance between the most lateral points on the acromion processes for each shoulder with a handheld tape measure (Lufkin, Sparks, Maryland) (Lockie et al., 2017). The 1RM for the TBP and CGBP were both assessed within the one session, the procedures of which will be described. The exercise that was completed first was randomized amongst the sample via the randomization function in a Microsoft Excel spreadsheet (Microsoft Corporation, Redmond, Washington). Participants refrained from intensive upper-body exercise and maintained their usual dietary intake in the 24-hour period prior to testing. This was done as restricted carbohydrate and fluid intake can negatively affect the performance of maximal strength exercises (Leveritt and Abernethy, 1999; Schoffstall et al., 2001). Additionally, participants were permitted to consume water as required throughout the testing session. No bench press suits, weightlifting belts, or other supportive garments were permitted during testing.

\section{RM TBP and CGBP Strength Testing}

The 1RM was measured for both the TBP and CGBP, and as noted the testing order for these lifts was randomized amongst the sample. An Olympic bar and weight plates (American Barbell, San Diego, CA) were used for both tests, which were conducted within a power rack (American Barbell, San Diego, CA). These methods describe the process if the 1RM TBP was completed first. The participants laid supine on a flat bench with their feet flat on the floor, and their head, shoulders and buttocks flat to the bench. Participants selected their 'strongest position' for the TBP (Lockie et al., 2017), and used a pronated grip. The distance between the index fingers on the bar was measured, and made relative to the participant's BAD. The distance was marked on the barbell with strips of athletic tape to ensure participants placed their hands on the bar at the same position for each repetition. The participant unracked the bar with assistance from a spotter if required, and began the lift with the arms extended and elbows locked (Gomo and 
Van Den Tillaar, 2016). The 'touch-and-go' procedure was utilized, in that the bar was required to touch the chest, pause briefly, before being pressed to full arm extension (Ware et al., 1995). A repetition was deemed to be successful when the bar was moved from the chest to a position of full elbow extension (Lockie et al., 2017). Failure to do this, or bouncing the bar off the chest (i.e. the bar was not controlled during the descent, and the ascent was assisted by a clear rebound off the chest), disqualified a repetition. A spotter was positioned for assistance with lift-off if required and for safety, but did not touch the bar except in the event of a failed lift (Clemons and Aaron, 1997). Participants initially completed $8-10$ repetitions at $50 \%$ of their estimated $1 \mathrm{RM}$, followed by 3-5 repetitions at $85 \%$ of the estimated 1RM, with 2 min recovery between sets (Lockie et al., 2017). Participants then completed a single repetition with $90 \%$ of the estimated 1RM, before completing their first $1 \mathrm{RM}$ attempt. If the participant was successful, loads increased by 2.5 $\mathrm{kg}$ until the participant failed. No more than five attempts were needed before the 1RM was reached. A 3-minute ( $\mathrm{min})$ rest was provided between attempts.

After a 10-min recovery period, participants completed the CGBP. The parameters that determined a successful lift were the same as those for the TBP, except for the grip width, which was $95 \%$ of BAD (Wagner et al., 1992). This placed the hands in a position inside the shoulders and close to the torso, which is similar to that required in upper-body pushing motions in sports (Cronin and Owen, 2004; Delextrat and Cohen, 2009; Stokes et al., 2010; Wheeler and Sayers, 2011), and thus was adopted in this study. As for the TBP, the grip width was marked on the barbell with athletic tape. The warm-up for the second test began by completing 3-5 repetitions at $85 \%$ of the participants' estimated 1RM, and then one repetition with 90\% 1RM (Gomo and Van Den Tillaar, 2016). Participants then completed their first 1RM attempt following a 3-min recovery period, and this process continued until the 1RM was attained.

Data was recorded during each $1 \mathrm{RM}$ attempt by a GymAware Powertool linear position transducer (Kinetic Performance Technology, Canberra, Australia), that produces reliable and valid data (Black, 2010; Drinkwater et al., 2007). The external end of the cable was attached to the inside of the barbell, and provided no resistance. The unit was placed on the floor directly underneath the bar, with the magnetic bottom positioned on a weight plate to ensure no movement during each lift. Velocity and bar movement was recorded at 50 Hertz; the load was entered into the software to calculate power and force, for every 3 millimeters of bar movement (Drinkwater et al., 2012). Further to this, within the software, an algorithm considered the angle of lift when deriving the variables in the vertical plane to ensure greater accuracy. Data was collected and stored on a $2^{\text {nd }}$ generation iPad (Apple Inc., Cupertino, California), before being uploaded to an online database (https://gymaware.kinetic.com.au) and passwordprotected account. Concentric variables were considered for the TBP and CGBP, and included: lift distance (bar displacement from lift initiation to lockout) in meters and duration in seconds (s); work (joules; J); and peak and mean power (watts; $\mathrm{W}$ ), force (newtons; $\mathrm{N}$ ), and velocity (meters per second; $\mathrm{m} \cdot \mathrm{s}^{-1}$ ). The mean values were derived over the duration of the concentric phase of each lift. The peak values were the highest number for each variable in the concentric phase.

\section{Statistical Analysis}

All statistics were computed using the Statistics Package for Social Sciences Version 24.0 (IBM, Armonk, United States of America). Descriptive statistics (mean $\pm \mathrm{SD}$ ) profiled each measured parameter. Stem-and-leaf plots were used to assess normality and find any outliers in the data (Lockie et al., 2017; Lockie et al., in press; Nimphius et al., 2016; Williamson et al., 1989). Outliers were treated via a winsorization method (Lockie et al., 2017; Lockie et al., in press), and this process ensured that parametric statistics could be used for this study. Accordingly, paired samples t-tests compared the variables between the TBP and CGBP, with significance set at $p<0.05$. Effect sizes $(d)$ were also calculated for the comparisons between the TBP and CGBP, where the difference between the means was divided by the pooled SD (Cohen, 1988). A $d$ less than 0.2 was considered a trivial effect; 0.2 to 0.6 a small effect; 0.6 to 1.2 a moderate effect; 1.2 to 2.0 a large effect; 2.0 to 4.0 a very large effect; and 4.0 and above an extremely large effect (Hopkins, 2004).

Pearson's two-tailed correlations determined 
relationships between the mechanical variables from the TBP with the CGBP, with significance again set at $p<0.05$. Correlations between all mechanical variables recorded in this study were completed, due to the interrelationship between factors such as the 1RM load, lift duration and lift distance, and the resulting work, and peak and mean power, force, and velocity in the TBP and CGBP (Lockie et al., 2017). Indeed, Lockie et al. (2017) highlighted how factors such as lift distance can influence peak and mean power, force, and velocity. The correlation strength was designated following the guidelines of Hopkins (2002). A correlation coefficient $(r)$ between 0 to 0.3 , or 0 to -0.3 , was considered small; 0.31 to 0.49 , or -0.31 to -0.49 , moderate; 0.5 to 0.69 , or -0.5 to 0.69 , large; 0.7 to 0.89 , or -0.7 to -0.89 , very large; and 0.9 to 1 , or -0.9 to -1 , near perfect for relationship prediction.

\section{Results}

The data for the TBP and CGBP is shown in Table 1. There was a significant difference between the grip width for the TBP and CGBP, with a very large effect. A greater load was lifted in the TBP, although this only had a small effect. Lift duration was longer for the TBP, and mean force was greater; however, both of these effects were small. Peak power, peak velocity, and mean velocity were greater for the CGBP, which had moderate, large, and small effects, respectively.

The correlations between the 1RM, lift distance, lift duration, and work from the TBP and all variables from the CGBP are displayed in Table 2. Correlations between peak and mean power, force and velocity from the TBP with all variables from the CGBP are shown in Table 3. The 1RM TBP correlated (moderate-to-near perfect relationships) with the CGBP 1RM, and peak and mean power and force. TBP lift distance and duration correlated (large-to-near perfect) with the same variables for the CGBP, as well as work. Work from the TBP correlated (moderateto-near perfect) with all variables from the CGBP, except for mean power and velocity. TBP peak power correlated (large-to-very large) with CGBP $1 R M$, lift distance, peak power, peak and mean force, peak velocity, and work. TBP mean power correlated (large-to-very large) with the same CGBP variables, and mean power. TBP peak and mean force related (large-to-near perfect) to CGBP $1 R M$, and peak and mean power and force. TBP peak velocity correlated with CGBP lift distance.

Table 1

Descriptive statistics (mean \pm SD) for grip width, one-repetition maximum (1RM) load, lift distance and duration, work, and peak and mean power, force, and velocity for the 1RM traditional bench press (TBP) and close-grip bench press (CGBP) in resistance-trained men $(n=20)$.

\begin{tabular}{cccccc}
\hline Variable & TBP & CGBP & $p$ value & $d$ & $d$ Strength \\
\hline Grip Width $(\mathrm{m})$ & $0.63 \pm 0.12$ & $0.35 \pm 0.03^{*}$ & $<0.001$ & 3.20 & Very Large \\
1RM Load $(\mathrm{kg})$ & $98.31 \pm 20.08$ & $92.47 \pm 19.14^{*}$ & $<0.001$ & 0.30 & Small \\
Lift Distance $(\mathrm{m})$ & $0.28 \pm 0.12$ & $0.31 \pm 0.14$ & 0.155 & 0.23 & Small \\
Lift Duration $(\mathrm{s})$ & $3.63 \pm 1.53$ & $2.93 \pm 0.94^{*}$ & 0.011 & 0.55 & Small \\
Work $(\mathrm{J})$ & $301.28 \pm 150.40$ & $270.85 \pm 131.36$ & 0.177 & 0.22 & Small \\
Peak Power $(\mathrm{W})$ & $350.03 \pm 85.18$ & $428.13 \pm 129.32^{*}$ & 0.001 & 0.71 & Moderate \\
Mean Power $(\mathrm{W})$ & $192.26 \pm 57.54$ & $214.85 \pm 79.87$ & 0.161 & 0.32 & Small \\
Peak Force $(\mathrm{N})$ & $1263.95 \pm 293.49$ & $1236.42 \pm 349.48$ & 0.452 & 0.09 & Trivial \\
Mean Force $(\mathrm{N})$ & $970.05 \pm 199.19$ & $912.24 \pm 184.63^{*}$ & $<0.001$ & 0.30 & Small \\
Peak Velocity $\left(\mathrm{m} \cdot \mathrm{s}^{-1}\right)$ & $0.35 \pm 0.07$ & $0.44 \pm 0.07^{*}$ & $<0.001$ & 1.29 & Large \\
Mean Velocity $\left(\mathrm{m} \cdot \mathrm{s}^{-1}\right)$ & $0.20 \pm 0.06$ & $0.23 \pm 0.06^{*}$ & 0.043 & 0.50 & Small \\
\hline
\end{tabular}

* Significantly $(p<0.05)$ different from the TBP. 


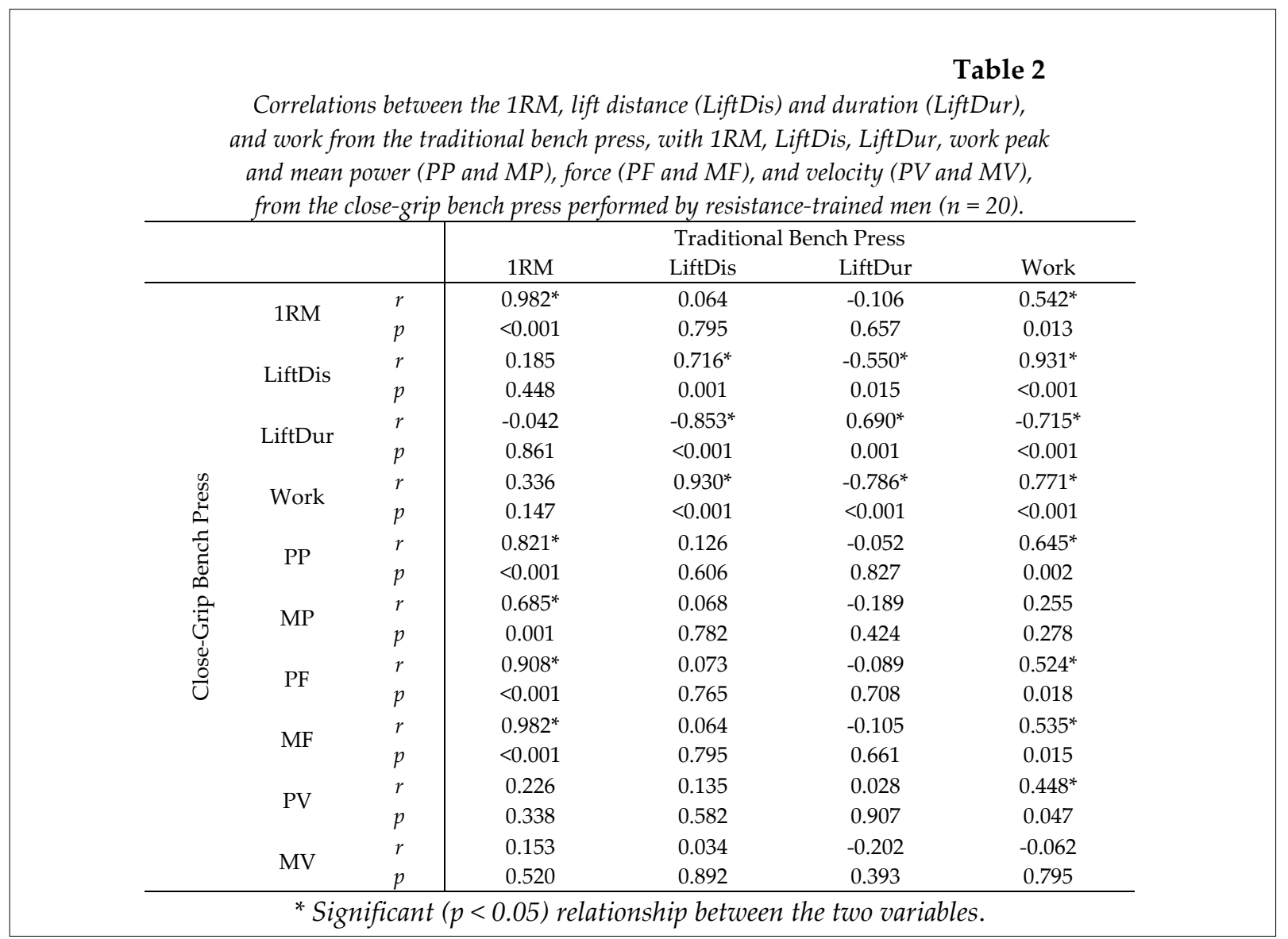

Table 3

Correlations between the peak and mean power (PP and MP), force (PF and MF), and velocity $(P V$ and $M V)$ from the traditional bench press, with $1 R M$,

lift distance (LiftDis) and duration (LiftDur), work, PP, MP, PF, MF, PV, and $M V$ from the close-grip bench press performed by resistance-trained men $(n=20)$.

\begin{tabular}{|c|c|c|c|c|c|c|c|c|}
\hline & & & \multicolumn{6}{|c|}{ Traditional Bench Press } \\
\hline & & & PP & MP & PF & MF & PV & MV \\
\hline \multirow{20}{*}{ 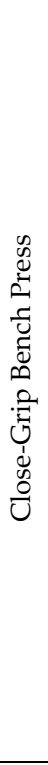 } & \multirow{2}{*}{$1 \mathrm{RM}$} & $r$ & $0.655^{*}$ & $0.536^{*}$ & $0.879^{*}$ & $0.980^{*}$ & -0.166 & -0.100 \\
\hline & & $p$ & 0.002 & 0.015 & $<0.001$ & $<0.001$ & 0.484 & 0.675 \\
\hline & \multirow{2}{*}{ LiftDis } & $r$ & $0.676^{*}$ & $0.525^{*}$ & 0.337 & 0.163 & $0.532^{*}$ & 0.372 \\
\hline & & $p$ & 0.001 & 0.021 & 0.158 & 0.504 & 0.019 & 0.117 \\
\hline & \multirow{2}{*}{ LiftDur } & $r$ & -0.396 & -0.346 & -0.113 & -0.015 & -0.390 & -0.297 \\
\hline & & $p$ & 0.084 & 0.135 & 0.636 & 0.949 & 0.089 & 0.203 \\
\hline & \multirow{2}{*}{ Work } & $r$ & $0.535^{*}$ & 0.301 & 0.394 & 0.311 & 0.229 & 0.061 \\
\hline & & $p$ & 0.015 & 0.197 & 0.085 & 0.182 & 0.331 & 0.797 \\
\hline & \multirow{2}{*}{ PP } & $r$ & $0.688^{*}$ & $0.484^{*}$ & $0.699^{*}$ & $0.816^{*}$ & 0.054 & -0.056 \\
\hline & & $p$ & 0.001 & 0.031 & 0.001 & $<0.001$ & 0.820 & 0.813 \\
\hline & \multirow{2}{*}{ MP } & $r$ & 0.411 & $0.532^{*}$ & $0.596^{*}$ & $0.689^{*}$ & -0.075 & 0.152 \\
\hline & & $p$ & 0.072 & 0.016 & 0.006 & 0.001 & 0.752 & 0.522 \\
\hline & \multirow{2}{*}{ PF } & $r$ & $0.704^{*}$ & $0.671^{*}$ & $0.890^{*}$ & $0.908^{*}$ & -0.018 & 0.089 \\
\hline & & $p$ & 0.001 & 0.001 & $<0.001$ & $<0.001$ & 0.940 & 0.708 \\
\hline & \multirow{2}{*}{$\mathrm{MF}$} & $r$ & $0.660^{*}$ & $0.545^{*}$ & $0.876^{*}$ & $0.980^{*}$ & -0.158 & -0.090 \\
\hline & & $p$ & 0.002 & 0.013 & $<0.001$ & $<0.001$ & 0.507 & 0.707 \\
\hline & \multirow{2}{*}{ PV } & $r$ & $0.461^{*}$ & 0.228 & 0.128 & 0.222 & 0.414 & 0.094 \\
\hline & & $p$ & 0.041 & 0.335 & 0.592 & 0.347 & 0.069 & 0.694 \\
\hline & \multirow{2}{*}{ MV } & $r$ & 0.078 & 0.370 & 0.125 & 0.161 & 0.078 & 0.376 \\
\hline & & $p$ & 0.745 & 0.108 & 0.600 & 0.499 & 0.744 & 0.102 \\
\hline
\end{tabular}

* Significant $(p<0.05)$ relationship between the two variables. 


\section{Discussion}

This study investigated relationships between mechanical variables for the TBP with the CGBP. This is the first study to conduct this type of analysis, as it is important to establish actual relationships between the mechanical variables for two lifts that can be used interchangeably by some practitioners (Green and Comfort, 2007; Haupt, 2001; Lockie et al., 2017), rather than relaying only on assumptions. If there were significant relationships between the two lifts, this could highlight some potential adaptations for the CGBP if it is used consistently in strength training, which is important as the hand position for this exercise more closely mirrors that used in the sporting environment (Cronin and Owen, 2004; Delextrat and Cohen, 2009; Stokes et al., 2010; Wheeler and Sayers, 2011). The results indicated that there were numerous significant relationships between important variables such as work, peak and mean power, and peak and mean force. These findings have important implications for coaches who program the CGBP in workouts for their athletes, as although this requires further analysis via a long-term training study, there could be similarities in strength adaptations experienced by the individuals who also utilize the CGBP in their training.

A significantly greater $1 R M$ load was lifted in the TBP, which translated to greater mean force, and is typical of previous research (Gomo and Van Den Tillaar, 2016; Lockie et al., 2017; Wagner et al., 1992). However, the effect for the difference in the 1RM load between the lifts was only small for the participants in the current study. There were positive relationships between the TBP 1RM load, and the CGBP 1RM load, and peak and mean power and force. In addition to this, there were positive relationships with peak and mean power and force for the TBP with the same variables in the CGBP. This means that those men who lifted a heavier load and generated greater force and power in the TBP also did so in the CGBP. This occurred even with the differences in technique and muscle activation in the CGBP (Barnett et al., 1995; Clemons and Aaron, 1997; Lehman, 2005). These findings are notable for individuals who utilize the CGBP. The CGBP could be programmed with the knowledge that if an individual can lift a heavy load and generate great force and power in the TBP, they should be able to do so in the CGBP. The strength adaptations that could result should also be more specific to the arm position used in the CGBP, which could have crossover into sports performance (Cronin and Owen, 2004; Delextrat and Cohen, 2009; Stokes et al., 2010; Wheeler and Sayers, 2011). Future research should investigate the actual strength adaptations that result from the consistent use of the CGBP.

Measuring the work completed during strength exercises is a valuable way to monitor the intensity and volume of resistance training (McBride et al., 2009). Thus, it is important to compare the relationships between the work completed in the TBP and CGBP. The results indicated that the TBP work had significant relationships with numerous CGBP variables. As work equates to force multiplied by distance, it would be expected that there would be positive correlations with lift distance and peak and mean force, and a negative relationship with lift time which was the case in this study. The further a load is moved during an exercise, the greater the work completed (Drinkwater et al., 2012). These results indicated that those men who did greater work in the TBP also did in the CGBP, which again highlights similarities between the two exercises.

There were few significant correlations between velocity from the TBP and CGBP. Additionally, CGBP peak velocity was significantly greater than the TBP, which would relate to the lower $1 \mathrm{RM}$. These results suggested that for a $1 R M$ lift, the velocity profile for the CGBP is different to the TBP. Gomo and Van Den Tillaar (2016) also found that male powerlifters generated a higher bar velocity with a 1RM bench press performed with a narrow grip $(\sim 0.39 \mathrm{~m})$ compared to lifts with wider hand positions $(\sim 0.57-0.75 \mathrm{~m})$. Lockie et al. (2017) found that strength-trained men and women also generated a greater peak bar velocity when performing a CBGP with a grip width of $95 \%$ BAD when compared to the TBP $(0.43 \pm 0.07 \mathrm{~m} \cdot \mathrm{s}-1$ vs. $0.35 \pm$ $0.06 \mathrm{~m} \cdot \mathrm{s}-1)$. As noted earlier, the explosive upperbody actions required in many sports involved the hands initiating the movement from close to the torso, before a forceful arm extension is completed. Improving the velocity associated with this action through the use of the CGBP 
could transfer to more effective passing in basketball (Delextrat and Cohen, 2009) and netball (Cronin and Owen, 2004), as the ball is commonly projected from the chest to a teammate. Enhanced movement velocity derived from training with the CGBP could also be beneficial for American football players, especially offensive lineman. Players from these positions often need to thrust their arms forcefully into their opponents to knock them off-balance when run or pass blocking, and the arms should be kept close to the torso to maintain leverage (Stokes et al., 2010). Lastly, fending in contact sports such as rugby and American football also involves fast and forceful extension of the arms into opponents to knock them off-balance (Wheeler and Sayers, 2011), for which training with the CGBP could be beneficial. Although confirmation is required from further investigation, the results from this study intimate that the CGBP could be used as a specific exercise to target higher velocity upperbody pushing movements for sport.

There are certain study limitations that should be acknowledged. Although the participants from this study were resistancetrained, they may not necessarily have been 'strong'. For example, the 1RM for the TBP in this study was $98.31 \pm 20.08 \mathrm{~kg}$; the male powerlifters in the study conducted by Gomo and Van Den Tillaar (2016) had a TBP of $131.5 \pm 22.9 \mathrm{~kg}$. Future research could investigate the mechanics of the CGBP in stronger populations, including powerlifters and athletes involved in pushing sports such as American football and rugby. Further investigations of the CGBP could also utilize motion capture to provide a more detailed analysis of the bar path and movement kinematics, and how that may relate to the TBP. These could also incorporate the use of electromyography (Golas et al., 2017; Stastny et al., 2017), as this would allow the analysis of any differences in muscle activation that occur in the 1RM CGBP when compared to the TBP. Nonetheless, the linear position transducer was used in this study because of its practical application, ease of use in the field, and provision of useful information for the strength and conditioning coach (Drinkwater et al., 2007; Harris et al., 2010). This illustrates usefulness for the data recorded in the current research. Future research could also investigate the relationship between multiple repetitions performed in the TBP and CGBP, as this could elucidate more information about potential mechanical adaptations that could result from these two exercises. Lastly, the effects that anthropometry could have on the mechanics of the TBP and CGBP should also be investigated, as this could influence relationships between the two lifts.

In conclusion, the results indicated that there were similarities in the mechanics of the TBP and the CGBP. Those men who lifted a greater TBP 1RM load and generated higher peak and mean power and force would likely do so in the CGBP as well. Greater work in the TBP also related to greater work in the CGBP. The strength adaptations that could result from the CGBP may be similar to those from the TBP, while also occurring from arm positions similar to those used in sport. However, the velocity profile for the CGBP was different to that of the TBP. This suggests that the CGBP could be used as a specific exercise to improve high-velocity, upper-body pushing movements. Future research should attempt to measure any long-term adaptations that occur with the consistent programming of the CGBP.

\section{Acknowledgements}

We would like to acknowledge our participants for their contribution to this study. Thanks also to Victoria Amran, Ibett Torne, Megan Beiley, Jillian Hurley, Michael Silva, and Karisha Mae Rosure for assisting with data collection. This research project received no external financial assistance. None of the authors have any conflict of interest.

\section{References}

Barnett C, Kippers V, Turner P. Effects of variations of the bench press exercise on the EMG activity of five shoulder muscles. J Strength Cond Res, 1995; 9: 222-227

Black M. Reliability and validity of the GymAware optical encoder to measure displacement data, 2010. 
Available at: http://www.kinetic.com.au/pdf/GA-Report2.pdf; accessed on 01.10.2016

Clemons JM, Aaron C. Effect of grip width on the myoelectric activity of the prime movers in the bench press. J Strength Cond Res, 1997; 11: 82-87

Cohen J. Statistical Power Analysis for the Behavioral Sciences (2nd ed.). Hillsdale, New Jersey: Lawrence Earlbaum Associates; 1988

Cronin JB, Owen GJ. Upper-body strength and power assessment in women using a chest pass. J Strength Cond Res, 2004; 18: 401-404

Delextrat A, Cohen D. Strength, power, speed, and agility of women basketball players according to playing position. J Strength Cond Res, 2009; 23: 1974-1981

Drinkwater EJ, Galna B, McKenna MJ, Hunt PH, Pyne DB. Validation of an optical encoder during free weight resistance movements and analysis of bench press sticking point power during fatigue. $J$ Strength Cond Res, 2007; 21: 510-517

Drinkwater EJ, Moore NR, Bird SP. Effects of changing from full range of motion to partial range of motion on squat kinetics. J Strength Cond Res, 2012; 26: 890-896

Faul F, Erdfelder E, Lang AG, Buchner A. G*Power 3: a flexible statistical power analysis program for the social, behavioral, and biomedical sciences. Behav Res Methods, 2007; 39: 175-191

Folland JP, Hawker K, Leach B, Little T, Jones DA. Strength training: isometric training at a range of joint angles versus dynamic training. J Sports Sci, 2005; 23: 817-824

Golas A, Maszczyk A, Pietraszewski P, Stastny P, Tufano JJ, Zajac A. Effects of pre-exhaustion on the patterns of muscular activity in the flat bench press. J Strength Cond Res, 2017; 31: 1919-1924

Gomo O, Van Den Tillaar R. The effects of grip width on sticking region in bench press. J Sports Sci, 2016; 34: 232-238

Green CM, Comfort P. The affect of grip width on bench press performance and risk of injury. Strength Cond J, 2007; 29: 10-14

Gross ML, Brenner SL, Esformes I, Sonzogni JJ. Anterior shoulder instability in weight lifters. Am J Sports Med, 1993; 21: 599-603

Harris NK, Cronin J, Taylor K-L, Boris J, Sheppard J. Understanding position transducer technology for strength and conditioning practitioners. Strength Cond J, 2010; 32: 66-79

Haupt HA. Upper extremity injuries associated with strength training. Clin Sports Med, 2001; 20: 481-490

Hopkins WG. A scale of magnitude for effect statistics, 2002. Available at: www.sportsci.org/resource/stats/index.html; accessed on 09.01.2016

Hopkins WG. How to interpret changes in an athletic performance test. Sportscience, 2004; 8: 1-7

Kitai TA, Sale DG. Specificity of joint angle in isometric training. Eur J Appl Physiol Occup Physiol, 1989; 58: 744-748

Lehman GJ. The influence of grip width and forearm pronation/supination on upper-body myoelectric activity during the flat bench press. J Strength Cond Res, 2005; 19: 587-591

Leveritt M, Abernethy PJ. Effects of carbohydrate restriction on strength performance. J Strength Cond Res, 1999; 13: 52-57

Lockie RG, Callaghan SJ, Moreno MR, Risso FG, Liu TM, Stage AA, Birmingham-Babauta SA, Stokes JJ, Giuliano DV, Lazar A, Davis DL, Orjalo AJ. An investigation of the mechanics and sticking region of a one-repetition maximum close-grip bench press versus the traditional bench press. Sports, 2017; 5: doi:10.3390/sports5030046

Lockie RG, Moreno MR, Lazar A, Risso FG, Tomita TM, Stage AA, Birmingham-Babauta SA, Torne IA, Stokes JJ, Giuliano DV, Davis DL, Orjalo AJ, Callaghan SJ. The one-repetition maximum mechanics of a high-handle hexagonal bar deadlift compared to a conventional deadlift as measured by a linear position transducer. J Strength Cond Res, in press: doi:10.1519/JSC.0000000000001781 
McBride JM, McCaulley GO, Cormie P, Nuzzo JL, Cavill MJ, Triplett NT. Comparison of methods to quantify volume during resistance exercise. J Strength Cond Res, 2009; 23: 106-110

Nimphius S, McGuigan MR, Suchomel TJ, Newton RU. Variability of a "force signature" during windmill softball pitching and relationship between discrete force variables and pitch velocity. Hum Mov Sci, 2016; 47: 151-158

Schoffstall JE, Branch JD, Leutholtz BC, Swain DE. Effects of dehydration and rehydration on the onerepetition maximum bench press of weight-trained males. J Strength Cond Res, 2001; 15: 102-108

Stastny P, Gołaś A, Blazek D, Maszczyk A, Wilk M, Pietraszewski P, Petr M, Uhlir P, Zając A. A systematic review of surface electromyography analyses of the bench press movement task. PLoS ONE, 2017; 12: e0171632

Stokes JV, Luiselli JK, Reed DD, Fleming RK. Behavioral coaching to improve offensive line pass-blocking skills of high school football athletes. J Appl Behav Anal, 2010; 43: 463-472

Wagner LL, Evans SA, Weir JP, Housh TJ, Johnson GO. The effect of grip width on bench press performance. Int J Sport Biomech, 1992; 8: 1-10

Ware JS, Clemens CT, Mayhew JL, Johnston TJ. Muscular endurance repetitions to predict bench press and squat strength in college football players. J Strength Cond Res, 1995; 9: 99-103

Wheeler KW, Sayers MGL. Rugby union contact skills alter evasive agility performance during attacking ball carries. Int J Sports Sci Coach, 2011; 6: 419-432

Williamson DF, Parker RA, Kendrick JS. The box plot: a simple visual method to interpret data. Ann Intern Med, 1989; 110: 916-921

\section{Corresponding author:}

\section{Dr. Robert Lockie}

California State University, Fullerton

Department of Kinesiology

800 N State College Blvd

Fullerton, CA 92831

USA

Phone (international): $\quad+1$ 657-278-4971

Email: rlockie@fullerton.edu 